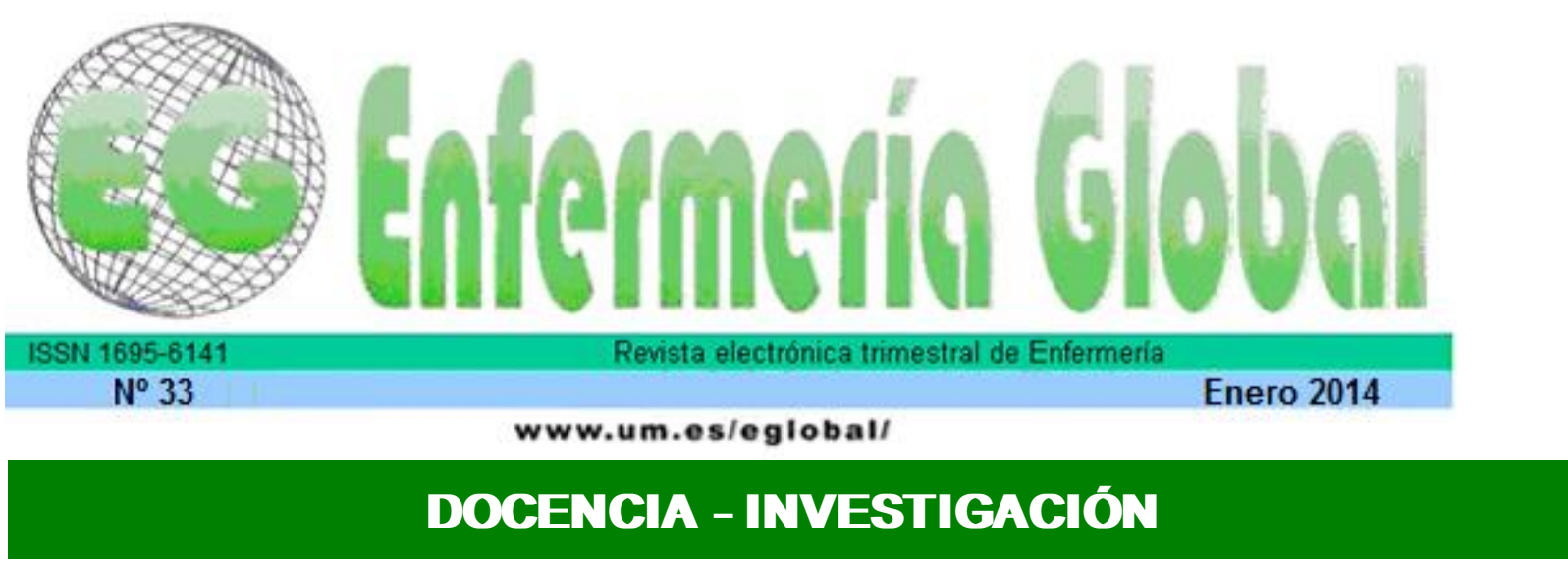

\title{
La autoestima como factor estresor intrapersonal para el consumo de alcohol en trabajadoras sexuales
}

Self-esteem as an intrapersonal stress factor on alcohol use in female prostitutes

\section{"Salinas Almaguer, Claudia Erika ${ }^{* *}$ Padilla Raygoza, Nicolás ${ }^{* * *}$ Ruiz Paloalto, $M^{a}$ Laura ****Vera Ramírez, Ana M ${ }^{a}$}

*Maestra en Ciencias de Enfermería, Profesora de la Facultad de Enfermería, Campus Minatitlán, Universidad Veracruzana ${ }^{* *}$ Doctor en Ciencias, Epidemiología, Departamento de Enfermería y Obstetricia, División de Ciencias de la Salud e Ingenierías, Campus Celaya Salvatierra, Universidad de Guanajuato. E-mail: raygosan@ugto.mx *** Doctora en Ciencias de Enfermería, Departamento de Enfermería Clínica, División de Ciencias de la Salud e Ingenierías, Campus Celaya Salvatierra, Universidad de Guanajuato ${ }^{* * * *}$ Maestra en Ciencias de Enfermería, Departamento de Enfermería Clínica, División de Ciencias de la Salud e Ingenierías, Campus Celaya Salvatierra, Universidad de Guanajuato. México.

Palabras clave: sexoservidoras; autoestima; consumo de alcohol

Keywords: Prostitutes; self-esteem; alcohol use/abuse

\section{RESUMEN}

Introducción. El alcoholismo es un problema mundial de salud y en las mujeres consumidoras se está incrementando. El sexo servicio podría estar ligado al consumo de alcohol.

Objetivo. Medir la relación entre autoestima como factor estresor intrapersonal y el consumo de alcohol en las trabajadoras sexuales.

Material y métodos. Estudio aprobado por el Comité de Investigación. Estudio transversal analítico en sexoservidoras en Cosoleacaque, Veracruz. Para consumo de alcohol se utilizó el cuestionario AUDIT y para la autoestima el inventario de Coopersmith.

Análisis estadístico. Se calculó Razón de Momios entre autoestima baja y consumo de alcohol, intervalos de confianza al 95\% y la Fracción Atribuible en los Expuestos. Se intentó generar un modelo de regresión logística.

Resultados. La muestra fueron 50 sexoservidoras. La Razón de Momios entre autoestima baja y consumo de alcohol fue de 5.08, con una Fracción Atribuible en Expuestos de 80.3\%. No se construyó el modelo logístico ya que ninguna variable de estudio mostró mejorar el modelo con el Test de Razón de Probabilidad. 
Conclusiones. De acuerdo con el modelo de los Sistemas de Betty Neuman, la autoestima baja tiene efecto e impacto en el consumo de alcohol.

\section{ABSTRACT}

Introduction. Alcoholism is a worldwide health issue, and female consumers are increasing. Prostitution could be linked to alcohol abuse.

Objective. To evaluate the effect of self-esteem as an intrapersonal stress factor on alcohol use in female prostitutes.

Material and methods. An approved study by the Research Committee. Cross-sectional, analytical study of prostitutes from Cosoleacaque, Veracruz, Mexico. For alcohol use the AUDIT questionnaire was used and for self-esteem, the Coopersmith inventory.

Statistical analysis. The Odds Ratio between low self-esteem and alcohol use, 95\% confidence intervals and Attributable Fraction in Exposed were calculated. An attempt was made to generate a logistic regression model.

Results. The trial sample was made up by 50 prostitutes. The Odds Ratio between low self- esteem and alcohol use was 5.08 (95\% Cl 0.59 a 43.95), and an Attributable Fraction in Exposed of $80.3 \%$. The logistic model was not built because no variable of the study demonstrated an improvement in the model using the probability ratio test.

Conclusions. According to Betty Neuman's system model, low self-esteem has a strong effect and impact in the use and abuse of alcohol.

\section{INTRODUCCIÓN}

El abuso de alcohol es un problema de salud en todo el mundo, de acuerdo con el Comité de Expertos de la Organización Mundial de la Salud (OMS), y la prevalencia del consumo es mayor en hombres, aunque se está incrementando su consumo entre las mujeres ${ }^{(1)}$.

El mayor consumo de alcohol se realiza en Europa Central y Asia Central, y posteriormente en América ${ }^{(1)}$ se clasifica en el quinto lugar entre los riesgos para la salud ${ }^{(2)}$. El segundo informe del Comité de Expertos de la OMS, señala que para el año 2002 , el alcohol provoca el $3.7 \%$ del total de defunciones y el $14.4 \%$ de la carga mundial de morbilidad ${ }^{(1)}$. Su costo directo representa 19,000 millones de dólares anuales al sistema de salud en los Estados Unidos.

El alcohol provoca dependencia, estimulación o depresión del Sistema Nervioso Central, ocasionando alucinaciones, trastornos de la función motora o del juicio del comportamiento ${ }^{(3)}$. El alcohólico afecta su vida, ocasionando problemas familiares e interpersonales que pueden causar ruptura del ciclo familiar; puede generar marginación social, accidentes de tránsito, problemas en el trabajo, desempleo y delitos, por lo que los costos sociales pueden ser mayores que los sanitarios ${ }^{(1)}$.

En México, la Encuesta Nacional de Adicciones (ENA) reporta que existían más de 32 millones de personas que consumían alcohol con edades entre 12 y 65 años y cerca de 13 millones lo consumieron el año anterior a la encuesta; los hombres bebedores representaron el $72 \%$, mientras que en las mujeres fue del $42.7 \%{ }^{(4)}$. Mariño, en la ciudad de México, reportó que el $60.7 \%$ de los hombre y $52 \%$ de las mujeres 
consumían alcohol todos los días y que en promedio las mujeres ingerían 4.2 copas por ocasión ${ }^{(5)}$.

En Valencia, España, las mujeres entrevistadas iniciaron el consumo del alcohol en edad temprana, y se desarrolló dependencia en forma progresiva, utilizando al alcohol para cubrir una realidad desagradable, llenar vacíos, aliviar tensiones; estas mujeres tenían sentimientos de culpa y baja autoestima, aislándose de su entorno, perdiendo la capacidad de relacionarse y dejando de cumplir las tareas del rol de mujeres ${ }^{(6)}$. En México, se reportó que las mujeres bebedoras, lo hacían debido a que el consumo de alcohol les producía sentimientos de felicidad, alegría, libertad; les hacía sentirse valientes para realizar actos desafiantes para mostrar su cuerpo, ya que, al consumir alcohol, se sentían atractivas; para otras, el beber, les permitía huir de su realidad, escapar de los problemas, pero al pasar el efecto del alcohol, tenían sentimientos de culpa ${ }^{(7)}$. Entre mujeres con alcoholismo, las que tuvieron mayor consumo del alcohol, refirieron baja autoestima ${ }^{(8)}$.

El trabajo sexual es una situación antigua, como la humanidad, y no existen cifras del número de mujeres que se dedican a este servicio a nivel mundial; tan sólo en Alemania se estimó que en el año 2005 existían 400,000 prostitutas ${ }^{(9)}$.

En Brasil, se reportó que el $70.7 \%$ de las sexoservidoras entrevistadas, consumían alcohol ${ }^{(10)}$; en Centroamérica durante el 2008, en mujeres sexoservidoras, se reportó que el $44 \%$ habían consumido más de 4 copas en al menos 4 ocasiones o más en los últimos 30 días y de ellas, el $26.9 \%$ dijeron haber consumido alcohol con su cliente más reciente ${ }^{(11)}$. En Colombia, se reportó que el $70.5 \%$ de las sexoservidoras consume alcohol, $56.8 \%$ lo hace ocasionalmente y el $13.65 \%$ en forma regular ${ }^{(12)}$.

En el sexoservicio es importante para incrementar el ingreso económico de la trabajadora, el inducir a los clientes a consumir alcohol, como se reportó en Colombia (13). La red de trabajadoras sexuales de América Latina y el Caribe, cuenta con 35 grupos de sexoservidoras en la República Mexicana, y calculan que en el Distrito Federal hay al menos 2,500 mujeres que ejercen el sexoservicio ${ }^{(14)}$.

El estado de Veracruz es la entidad que cuenta con más personas dedicadas al trabajo sexual, con 13,278 personas, de las cuales 11,991 son mujeres ${ }^{(15)}$.

Según el modelo de Betty Neuman, el individuo como sistema puede verse afectado por factores estresores intrapersonales, extrapersonales e interpersonales; la autoestima se considera un factor estresor intrapersonal, variable psicológica, que puede afectar al sistema, llevándola a un estado de desestabilidad, produciendo como reacción el consumo de alcohol ${ }^{(16)}$.

Las trabajadoras sexuales representan un grupo vulnerable de la población femenina, ya que debido al oficio que ejercen, son marginadas; no cuentan con programas de salud integral, pues sólo se les realizan exámenes mensuales de enfermedad de transmisión sexual y VIH/SIDA ${ }^{(17)}$.

El objetivo fue medir el efecto e impacto de la autoestima baja sobre el consumo de alcohol, en trabajadoras sexuales de Cosoleacaque. 


\section{MATERIAL Y MÉTODOS}

El estudio fue aprobado por el Comité de Investigación de la División de Ciencias de la Salud e Ingenierías del Campus Celaya Salvatierra de la Universidad de Guanajuato y autorización por el $\mathrm{H}$. Ayuntamiento de Cosoleacaque, Veracruz en México.

Tipo de estudio. Fue un estudio transversal analítico, basado en la comunidad.

Sujetos de estudio. Sexoservidoras registradas en el Municipio de Cosoleacaque, Veracruz, México, siendo un total de 98.

Criterios de inclusión. Mujeres que se dediquen al sexoservicio, de 18 o más años, y que acepten por escrito participar.

Criterios de exclusión. Sexoservidoras embarazadas.

Muestra. Se invitó a las 98 sexoservidoras a participar en el estudio, de las cuales aceptaron $53(58.89 \%)$ y sólo estuvieron en la entrevista 50 (51.02\%).

Procedimientos. Se midieron las variables: edad, en años cumplidos; la escolaridad en años estudiados a partir de la educación elemental; ingreso económico en pesos mexicanos mensuales; la edad en que ingirió la primera copa de alcohol; el número de hijos que habían procreado las mujeres estudiadas y el tiempo en meses de dedicarse al sexoservicio.

Para conocer el consumo de alcohol, se utilizó el Test de Identificación de los Trastornos debidos al Consumo de Alcohol (AUDIT) diseñado por la OMS en 1992, que fue utilizado para identificar personas con un patrón de consumo de alcohol de riesgo o perjudicial o dependencia al alcohol ${ }^{(18)}$; se clasificaron como consumidoras si tuvieron un puntaje de 8 a 40 y como no consumidoras de 0 a 7 . Es un instrumento de 10 ítems con respuestas en escala con puntaje de 0 a 4 . Se ha medido su confiabilidad en población mexicana con una Alpha de Cronbach de 0.87 para la escala total.

Para conocer el nivel de autoestima se utilizó el inventario de autoestima de Coopersmith, con una confiabilidad de Alpha de Cronbach de 0.81 en población mexicana. Consta de 25 reactivos con respuestas dicotómicas; se clasificaron como autoestima baja con 0 a 10 puntos, autoestima media de 11 a 22 puntos ${ }^{(19)}$.

Tamaño de muestra. Esperando que el $80 \%$ de los consumidoras de alcohol tuvieran autoestima baja y $55 \%$ de las no consumidoras de alcohol tuvieran autoestima baja, con una Razón de Momios (RM) esperada de 3.2, con $95 \%$ de precisión y $80 \%$ de poder, el tamaño mínimo de muestra es de 16 consumidoras de alcohol y 16 no consumidoras de alcohol (Epidat 3.1, 2008, Xunta de Galicia y Organización Panamericana de la Salud).

Análisis estadístico. Se utilizó estadística descriptiva para las variables sociodemográficas; para conocer el efecto de la autoestima sobre el consumo de alcohol, se calculó RM intervalos de confianza al $95 \%$ y para conocer el impacto de la autoestima baja sobre el consumo de alcohol, se calcula la Fracción Atribuible en expuestos (FAE). 
Se intentó construir un modelo de regresión logística entre consumo de alcohol, autoestima, y las variables sociodemográficas, usando el Test de Razón de Probabilidad (TRP) para conocer si el modelo se mejora con más variables o no.

Para el análisis estadístico se utilizó STATA 10.1 (StataCorp LP, College Station, TX, EUA).

\section{RESULTADOS}

La muestra estuvo constituida por 50 sexoservidoras de 98 registradas en el Municipio de Cosoleacaque, Veracruz.

En la tabla I se muestran las características categóricas de las sexoservidoras participantes. Predominaron las solteras (62\%), que no tenían otra ocupación (40\%), y las que profesaban la religión católica $(66 \%)$.

Tabla I Características categóricas sociodemográficas de la muestra, Cosoleacaque, Ver., 2011 $(n=50)$

\begin{tabular}{|l|l|l|}
\hline Variable & $\mathbf{N}$ & $\%$ \\
\hline Estado civil & 31 & 62.00 \\
Soltera & 8 & 16.00 \\
Casada & 8 & 6.00 \\
Divorciada & 3 & 12.00 \\
Separada & 6 & 4.00 \\
Unión libre & 2 & \\
\hline Ocupación & & 2.00 \\
Estudiante & 1 & 36.00 \\
Ama de casa & 48 & 8.00 \\
Empleada & 7 & 14.00 \\
Comerciante & 20 & 40.00 \\
Ninguno & 33 & 66.00 \\
\hline Religión & 17 & 34.00 \\
Católica & Ninguna & \\
\hline
\end{tabular}

Fuente: Cuestionario del estudio

Las características cuantitativas de la muestra se reportan en la tabla II. El promedio de edad fue de 31 años, con escolaridad de 6 años, con ingreso económico de 2900 pesos mexicanos mensuales (260 dólares), a los 21 años en promedio consumieron su primera copa de alcohol, con dos hijos en promedio y 29 meses de dedicarse al sexoservicio.

Tabla II Características discretas de los integrantes de la muestra, Cosoleacaque, Ver. $2011(n=50)$.

\begin{tabular}{|l|l|l|}
\hline Variable & Rango & Media \pm s \\
\hline Edad (años) & 18 a 60 & $31.04 \pm 8.5$ \\
\hline Escolaridad (años estudiados) & 1 a 12 & $6.16 \pm 3.10$ \\
\hline Ingreso económico mensual & 800 a 8000 & $2900 \pm 1490.80$ \\
\hline $\begin{array}{l}\text { Edad cuando ingirió la primera copa de } \\
\text { alcohol (años) }\end{array}$ & 9 a 48 & $21.5 \pm 7.20$ \\
\hline
\end{tabular}




\begin{tabular}{|l|l|l|}
\hline Número de hijos & 0 a 5 & $2.20 \pm 1.21$ \\
\hline $\begin{array}{l}\text { Tiempo de dedicarse al sexoservicio } \\
\text { (meses). }\end{array}$ & 1 a 120 & $29.94 \pm 30.08$ \\
\hline
\end{tabular}

Fuente: Cuestionario del estudio.

En la tabla III se tabulan los diagnósticos de consumo de alcohol y de categoría de autoestima. Se obtiene una $\mathrm{RM}=5.08$ con intervalos de confianza al $95 \%$ de 0.59 a 43.95 y la Fracción Atribuible en Expuestos $=80.3 \%$; por lo tanto, hay un importante efecto de la autoestima baja sobre el consumo de alcohol y la AFE muestra que el $80.3 \%$ de las consumidoras de alcohol. no lo hubieran hecho si su autoestima fuera media 0 alta.

Tabla III Tabulación entre autoestima y consumo de alcohol, Cosoleacaque, $2011(n=50)$

\begin{tabular}{|l|ll|ll|l|l|}
\hline & \multicolumn{9}{|l|}{ AUDIT } \\
\hline COOPERSMITH & \multicolumn{2}{|l|}{ Consumo de alcohol } & \multicolumn{2}{l|}{ No consumo de alcohol } & \multicolumn{2}{l|}{ Total } \\
& $\mathrm{n}$ & $\%$ & $\mathrm{n}$ & $\%$ & $\mathrm{n}$ & $\%$ \\
\hline Autoestima baja & 11 & 22.00 & 1 & 2.00 & 12 & 24.00 \\
\hline $\begin{array}{l}\text { Autoestima } \\
\text { media }\end{array}$ & 26 & 52.00 & 12 & 24.00 & 38 & 76.00 \\
\hline Total & 37 & 74.00 & 13 & 26.00 & 50100.00 \\
\hline
\end{tabular}

Fuente: Cuestionarios AUDIT y Coopersmith

Se generó un modelo de regresión logística entre autoestima baja y consumo de alcohol, agregando edad (TRP, $\mathrm{p}>0.05$ ), ocupación (TRP, $\mathrm{p}>0.05$ ), religión (TRP, $p>0.05$ ), escolaridad (TRP, $p>0.05$ ), ingreso económico mensual (TRP, $p>0.05$ ), tiempo de dedicarse al sexoservicio (TRP, $p>0.05$ ), número de hijos (TRP, $p>0.05$ ), por lo que ninguna de estas variables actúan como confusores ni modificadores de efecto.

\section{DISCUSIÓN}

La muestra estuvo integrada por 50 sexoservidoras de un universo de 98 trabajadoras sexuales, registradas en el $\mathrm{H}$. Ayuntamiento de Cosoleacaque, Veracruz.

En la muestra del estudio, $14 \%$ se dedicaban al comercio y $8 \%$ eran empleadas, además de dedicarse al sexoservicio, resultados similares al estudio de Colombia, en mujeres de sexoservicio prepago, donde el 34.9\% estaban empleadas, además del sexoservicio ${ }^{(12)}$; en este estudio $86.4 \%$ eran solteras y en el estudio de Colombia, lo era el $62 \%$ de la muestra ${ }^{(12)}$.

En Brasil, se reportó que $84 \%$ de mujeres consumidoras de alcohol eran católicas y el $77 \%$ tenían hijos ${ }^{(20)}$, y en la muestra en Cosoleaque, 66\% profesaban la religión católica y $76 \%$ tenían hijos (Tabla I).

En cuanto a la edad de consumo de la primera copa de alcohol, los resultados obtenidos en la muestra de sexoservidoras de Cosoleacaque, con rango de 9 a 48 años (Tabla II), concuerda con lo reportado por la Encuesta Nacional de Adicciones $^{(21)}$, donde se señala que el inicio del consumo de alcohol es durante la adolescencia. 
La RM de 5.08, indica que las consumidoras de alcohol tenían cinco veces más probabilidad de tener autoestima baja que las que no consumían alcohol (Tabla III), indicando que la autoestima baja es un factor estresor intrapersonal que ayuda a que se consuma alcohol.

Las personas con autoestima baja esperan ser engañadas, menospreciadas; como defensa se escudan en la desconfianza, llevándolas a la soledad y aislamiento, dando lugar a apatía, indiferencia ente sí mismas y ante los demás y pueden refugiarse en el consumo de alcohol ${ }^{(22)}$.

Entre las sexoservidoras se han reportado los rasgos y características psicológicas de familia poco estructurada, ausencia de modelos de identificación, sentimiento de abandono a edad temprana, privación temprana de afecto, sentimientos de minusvalía, inseguridad e inferioridad, dando lugar a dificultad para establecer relaciones interpersonales satisfactorias, estado de frustración ante la vida, sensibilidad al rechazo, sentimientos de culpa por dedicarse al sexoservicio y consumo de alcohol como inhibidor de sus sentimientos de insatisfacción y malestar $^{(17)}$.

De acuerdo al modelo de Neuman, la autoestima baja pudiera estar atravesando las líneas de defensa del sistema, generando desestabilidad, provocando como reacción el consumo de alcohol, creando un ambiente de protección de la realidad que viven ${ }^{(16)}$.

\section{CONCLUSIÓN}

De acuerdo al modelo de los Sistemas de Betty Neuman, la autoestima baja está actuando como un factor estresor intrapersonal para el consumo de alcohol en las trabajadoras sexuales.

Se recomendaría realizar estudios epidemiológicos incluyendo otros factores estresores intra y extrapersonales, como el hecho de que inducir el consumo de alcohol entre los clientes, incrementa su ingreso económico.

\section{REFERENCIAS BIBLIOGRÁFICAS.}

1.- Comité de Expertos de la OMS en problemas relacionados con el consumo de alcohol. Segundo Informe, 2007, Organización Mundial de la Salud (http://who.int/substance abuse/expert committee alcohol trs944 spanish.pdf)

(Acceso el 18 Julio 2012).

2.- Harris C. El problema del alcohol. Perspectivas de salud, 2005; 10(1):19-23 (http://www.paho.org/spanish/dd/pin/Numero21 articulo04.htm) (Acceso 18 Julio 2012).

3.- Avila JJ, Gonzalez D. Diferencias de género en la enfermedad alcohólica. Adicciones, 2007;19(4):383-392 (http://www.adicciones.es/files/avila.pdf) (Acceso 18 Julio 2012).

4.- Instituto Mexicano de Psiquiatría. Encuesta Nacional de Adicciones-ENA 2002. (http://www.consulta.com.mx/interiores/99 pdfs/15 otros pdf/ENA.pdf) (Acceso 18 Julio 2012).

5.- Mariño MC, Berenson S, Medina ME. Sindrome de dependencia al alcohol: comparación entre hombres y mujeres. Salud Mental, 2005;28(3):33-39. 
(http://www.medigraphic.com/pdfs/salmen/sam-2005/sam054d.pdf) (Acceso 18 Julio 2012).

6.- Gomez J, Valderrama JC, Girva T, Tortajada S, Guillot J. Mujeres dependientes del alcohol o cocaína: un estudio comparativo desde una perspectiva cualitativa. Adicciones, 2008;20(3):237-244 (http://www.adicciones.es/files/gomez\%20moya.pdf) (Acceso 18 Julio 2012).

7.- Cortaza L, Villar MA. Surge en mi otra mujer: significados del consumo de alcohol en mujeres mexicanas. Esc Anna Nery Rev Enferm, 2008;12(4):693-698 (http://www.eean.ufri.br/revista enf/20084/11-ART\%20.pdf) (Acceso 18 Julio 2012).

8.- Alonso MM, Alvarez J, Lopez KS, Guzman FR, Martinez R. Factores personales, psicosociales y consumo de alcohol en mujeres adultas. Anuario de Investigación en Adicciones, 2008;9(1). (http://webcache.googleusercontent.com/search?q=cache:http://www.cucs.udg.mx/avi sos/anuario investigacion adicciones/Anuario9/2.htm) (Acceso 18 Julio 2012).

9.- DW-worldwide. Deutsche Welle (http://www.dwworld.de/dw/article/0,1772881,00.html) (Acceso 18 Julio 2012).

10.- Schreiner L, Ludwig L, Ramos F, Cunha EV, Marilio D, Lopez C, Coutinho M, Madeira T, Rosa N, Picon P. Prevalencia de sintomas depressivos em uma amostra de prostitutas de Porto Alegre. R Psiquiatr. RS, 2004; 26(1): 13-20 (http://www.scielo.br/pdf/rprs/v26n1/20472.pdf) (Acceso 18 Julio 2012).

11.- Galvan G, Armero J, Paz G, Creswell J. Encuesta centroamericana de vigilancia de comportamiento sexual y prevalencia de VIH/ITS en poblaciones vulnerables. Ministerio de Salud El Salvador, 2008:pp.73 (http://www.pasca.org/sites/default/files/modelo ecvc hsh ts pvvx.pdf) (Acceso $18 \mathrm{de}$ Julio 2012).

12.- Bermúdez A, Gaviria AM, Fernández H. estilos psicológicos de la personalidad de un grupo de mujeres adultas jóvenes dedicadas a la prostitución prepago en la ciudad de Medellín. Terapia psicológica, 2007; 25(1):25-37 (http://redalyc.uaemex.mx/src/inicio/ArtPdfRed.jsp?iCve=78525102) (Acceso 18 de Julio 2012).

13.- Lafaurie MM, Gomez PI, Bernal AJ, Gualteros MA, Hassan VP, Lozano JA, Tovar CV. Embarazo en mujeres en situación de prostitución: autocuidado y prácticas de riesgo. Estudio cualitativo. Rev Colomb Obstet Ginecol, 2008;59(4):285-296 (http://www.scielo.org.co/scielo.php?pid=S0034-

74342008000400004\&script=sci arttext\&ting es) (Acceso 19 Julio 2012).

14.- Escuela de Posgrado. Universidad Nacional de San Martín. Ni una mujer más en las redes de prostitución. (http://www.unsam.edu.ar/escuelas/humanidades/centros/cedehu/material/trata225.pdf) (Acceso 19 Julio 2012).

15.- Sagayo F. Veracruz con más sexoservicio. Diario del Itsmo 2009.

16.- Marriner A, Raile. Modelos y teorías de enfermería. 6aㅡ Ed., Madrid, Elsevier Mosby, 2007;p. 317-336.

17.-Romero L, Quintanilla A. Prostitución y drogas. Estudio psicosociológico de la prostitución en México y su relación con la farmacodependencia. México, Editorial Trillas, 1993: pp.19.

18.- Babor TF, Higgins JC, Saunders JB, Monteiro MG. AUDIT Cuestionario de identificación de los trastornos debidos al consumo de alcohol. Pautas para su utilización en Atención Primaria. Ginebra, Organización Mundial de la Salud, 2001. (http://www.who.int/substance abuse/activities/en/AUDITmanualSpanish.pdf)

(Acceso 19 Julio 2012). 
19.- Lara MA, Verduzco MA, Acevedo M, Cortés J. Validez y confiabilidad del inventario de autoestima de Coopersmith para adultos, en población mexicana. Rev Latinoamericana de Psicología, 1993;25(2): 247-255.

(http://redalyc.uaemex.mx/src/inicio/ArtPdfRed.jsp?iCve=80525207\&iCveNum=9975) (Acceso 19 Julio 2012).

20.- Nobrega MPSS, OliveiraEM. Mulheres usuárias de álcool: análise qualitativa. Rev Saude Publica, 2005;39(5): 816-823.

21.- Instituto Nacional de Salud Pública. Encuesta Nacional de Adicciones-ENA 2008.

Cuernavaca, Instituto Nacional de Salud Pública, 2008 (http://www.conadic.salud.gob.mx/pdfs/ena08/ENA08 NACIONAL.pdf) (Acceso 19 Julio 2012).

22.- Rodríguez M, Pellicer G, Domínguez M. Autoestima: clave del éxito personal. $2^{\underline{a}}$ ed., México, El Manual Moderno, 1988;p.9-29. 